\title{
Quantifying Local Structure of Complex Oxides Using Accurate and Precise Scanning Transmission Electron Microscopy
}

James M. LeBeau ${ }^{1}$, Matthew Cabral ${ }^{1}$, J. Houston Dycus ${ }^{1}$, Everett Grimley ${ }^{1}$, Shujun Zhang $^{2}$, Elizabeth Dickey $^{1}$

1. Department of Materials Science \& Engineer, North Carolina State University, Raleigh NC USA

2. Institute for Superconducting \& Electronic Materials, University of Wollongong, Wollongong Australia

Quantifying local structure is critical to understand and control material properties. While pair distribution function techniques from diffraction are commonly used to investigate the average local structure of a material, these approaches are limited by the lack of chemically and spatially resolved details. While electron microscopy exhibits superior spatial resolution, accurate determination of structure has been limited by imprecise knowledge of the illuminating and diffraction conditions (parallel beam) or sample drift (STEM). In contrast, the revolving STEM (RevSTEM) method introduces scan rotation between a series of fast-acquisition STEM images to encode the information about sample drift and leads to near distortion-free imaging after post-processing [1].

In this talk, we will discuss how RevSTEM imaging can be made highly accurate and precise for local structure determination. Beyond drift, we will also show that the scan system axes are not orthonormal, resulting a 'global residual distortion' which must be calibrated to achieve accuracy [2]. We will demonstrate and explain how to accurately and precisely remove this distortion. This is achieved using measurement of the projected-PDF (P-PDF) by locating and calculating the distance between all $\mathrm{n}^{\text {th }}$ nearest neighbors (Figure 1a) [3]. We will show how the standard deviation of each $n^{\text {th }}$ near neighbor distance can then be used to determine the coefficients of the calibration transformation matrix (Figure $1 b)$ :

$$
T=\left(\begin{array}{cc}
1 & 1 \\
x_{1} & 1+x_{2}
\end{array}\right)
$$

Using well-calibrated RevSTEM imaging, we will then show how this approach opens new avenues to characterize local structure in complex oxide materials. Multiple case studies for complex oxides will be presented, including thin film and bulk materials [4]. For example, we will show how picometer precise measurements enable the capability to directly observe static atomic displacements within a complex oxide solid solution exhibiting differences in local bonding (Figure 2a). We will also explore how local deviations from cubic symmetry can be observed in the oscillations of the standard deviations from the PPDF (Figure 2b). Finally, we will discuss the application of application of spatial statistics, such as Bayesian classifiers, to explore the connection between local chemistry and structure [5].

\section{References:}

[1] Sang, X. and LeBeau, J. M. Ultramicroscopy 138, (2014) $28 .$.

[2] Dycus, J. H., et al. Microscopy and Microanalysis 21, (2015) $946 .$.

[3] Sang, X., Oni, A. A., and LeBeau, J. M. Microscopy and Microanalysis 20, (2014) 1764.

[4] Sang, X., et al, J. M. Applied Physics Letters 106, (2015, 61913. 
[5] The authors acknowledge funding from the National Science Foundation (DMR-1608656), the Air Force Office of Scientific Research (FA9550-14-1-0182), and the NSF funded Center of Dielectrics and Piezoelectrics (IIP-1361503). The authors acknowledge the use of the Analytical Instrumentation Facility at NCSU, which is supported by the state of North Carolina and the National Science Foundation.
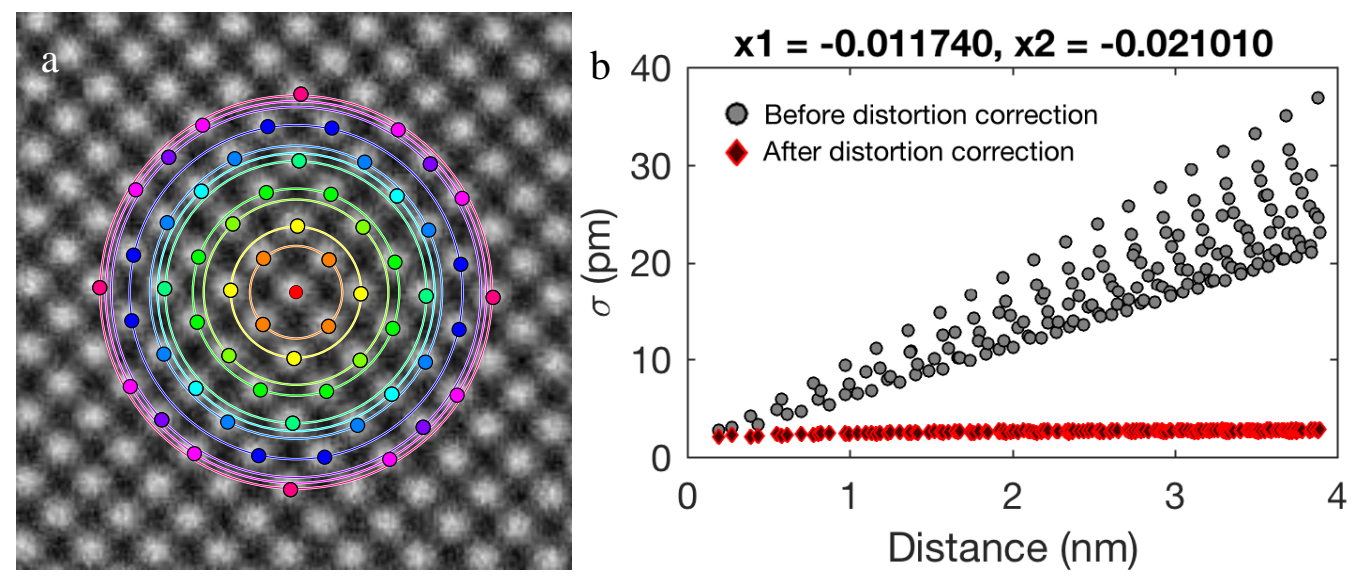

Figure 1. (a) Determination of $\mathrm{n}^{\text {th }}$ nearest neighbors across an image of $\langle 100\rangle$ Si provide a data set to accurately calibrate for residual scan system distortion. (b) The standard deviation of $\mathrm{n}^{\text {th }}$ nearest neighbor distances before and after distortion correction.
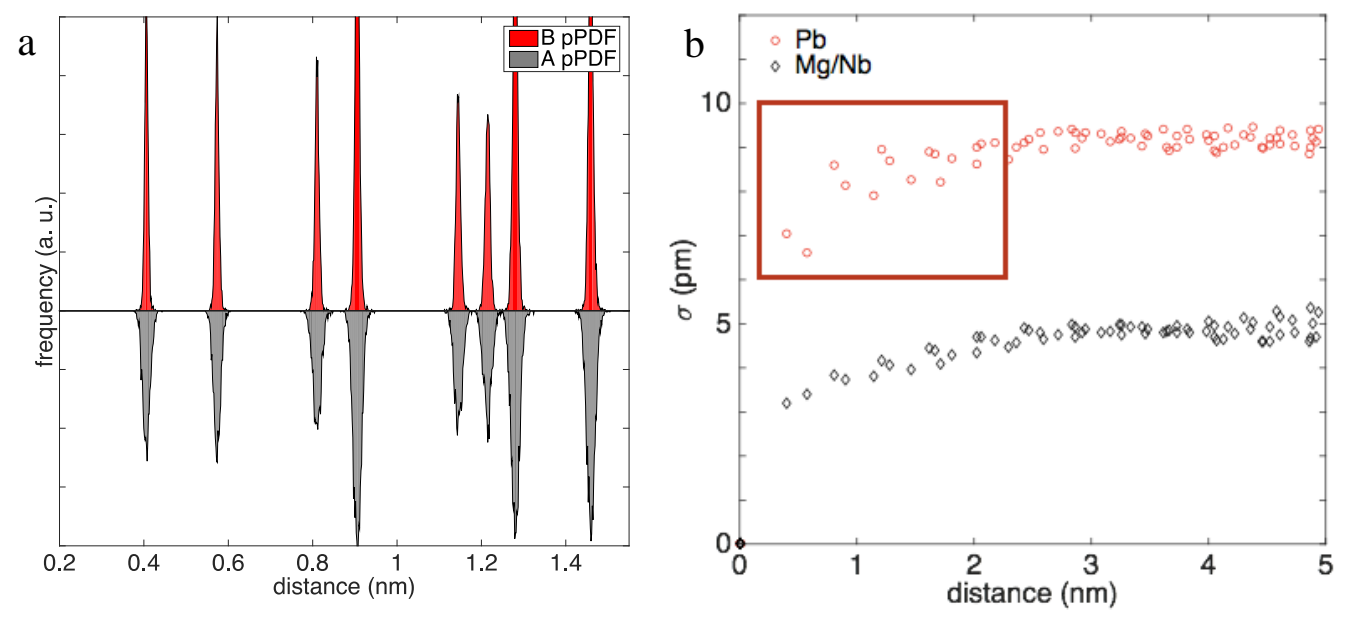

Figure 2. (a) Projected PDF measurements provide direct access to local crystallographic information across an image and, unlike diffraction based PDF methods, can readily be separated for each sublattice. (b) The standard deviation of $\mathrm{n}^{\text {th }}$ nearest neighbor distances after distortion correction highlighting residual oscillations in $\mathrm{PbMg}_{1 / 3} \mathrm{Nb}_{2 / 3} \mathrm{O} 3$ that result from local deviations from cubic symmetry. 